Supporting information

\title{
Fabrication of Covalent SAM/Au Nanoparticle/Boron-Doped Diamond Configurations with a Sequential Self-Assembly Method
}

Takeshi Kondo, ${ }^{\dagger}$ Shinsuke Aoshima, ${ }^{\dagger}$ Kensuke Honda, ${ }^{\ddagger}$ Yasuaki Einaga, ${ }^{\S}$ Akira Fujishima, ${ }^{\text {Il }}$ and Takeshi Kawai* ${ }^{* \dagger}$

${ }^{\dagger}$ Department of Industrial Chemistry, Faculty of Engineering, Tokyo University of Science, 1-3 Kagurazaka, Shinjuku-ku, Tokyo, 162-8601 Japan, 'Department of Chemistry and Earth Sciences, Faculty of Science, Yamaguchi University, 1677-1 Yoshida, Yamaguchi-shi, Yamaguchi 753-8512 Japan, ${ }^{\S}$ Department of Chemistry, Faculty of Science and Technology, Keio University, 3-14-1 Hiyoshi, Yokohama, 223-8522 Japan, "Kanagawa Academy of Science and Technology (KAST), 3-2-1 Sakado Takatsu-ku, Kawasaki, Kanagawa, 213-0012 Japan 

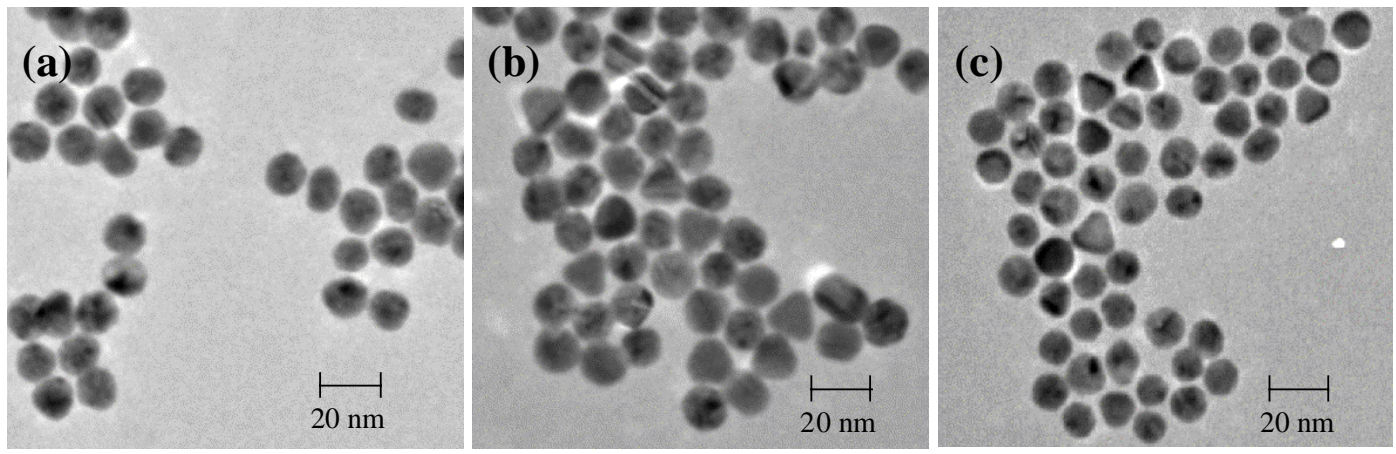

Figure S1. TEM images of (a) c-AuNP, (b) MAA-AuNP and (c) DODAC-AuNP.

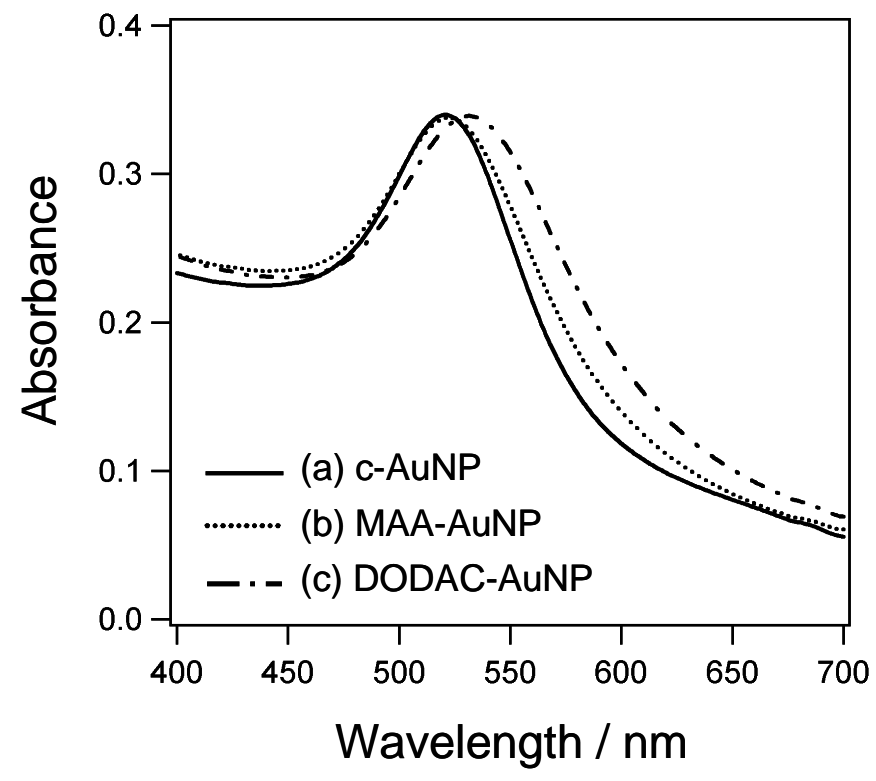

Figure S2. Absorption spectra for (a) c-AuNP, (b) MAA-AuNP, and (c) DODAC-AuNP solutions. 